\title{
Examination of Transepithelial Exchange of Water and Solute in the Rat Renal Pelvis
}

\author{
Joanne Bargman, Sharon L. Leonard, Elizabeth McNeely, \\ Channing Robertson, and Rex L. Jamison \\ Division of Nephrology, Department of Medicine, Department of \\ Chemical Engineering, Stanford University, \\ Stanford, California 94305
}

bstract. Severance of the ureter beyond the renal papilla causes a fall in urinary osmolality, which suggests that exchange of water or solute between urine and renal parenchyma normally occurs in the intact renal pelvis. We examined water and solute flux in the renal pelvis with micropuncture and microcatheterization techniques. Four groups of antidiuretic rats were studied. Group I $(n=17)$ underwent micropuncture through the intact contracting ureter. Urine samples were obtained at the papillary tip, and in the pelvis beside the base of the extrarenal papilla. Urinary osmolality at the base, $880 \pm 97 \mathrm{mosmol} / \mathrm{kg} \mathrm{H}_{2} \mathrm{O}$ (mean $\pm \mathrm{SE}$ ), was less than that at the tip, $1,425 \pm 104 \mathrm{mosmol} / \mathrm{kg} \mathrm{H} \mathrm{H}_{2} \mathrm{O}(P<0.005)$. In group II $(n=24)$, samples were analyzed for inulin and osmolality. In 15 rats (group IIA), comparison was made between base and tip samples. In the other nine animals (group IIB), comparisons were made among base, tip, and bladder samples and urea was also measured. In group II (A and B combined) urine-to-plasma (U/P) osmolality was lower at the base, $4.31 \pm 0.27$, than at the tip, 6.08 $\pm 0.23(P<0.001)$, and U/P inulin was lower at the base, 192 \pm 25 , than at the tip, $306 \pm 16$ ( $P$ $<0.001)$. In group IIB, the bladder urine had a lower $\mathrm{U} / \mathrm{P}$ osmolality, $5.27 \pm 0.25$, than the tip, $6.01 \pm 0.31(P$ $<0.02$ ). The U/P urea was $59 \pm 10.6$ (base), $98 \pm 9.4$ (tip) (base vs. tip, $P<0.05$ ), and $81 \pm 6.5$ (bladder, $P<0.005$, compared with tip). In group III $(n=8)$, samples were

A preliminary report of these findings has been published in abstract form: Leonard, S., and R. Jamison. 1983. Clin. Res. 31:516A.

Dr. Bargman is a Fellow of the Medical Research Council of Canada.

Received for publication 8 August 1983 and in revised form 19 July 1984.

J. Clin. Invest.

(c) The American Society for Clinical Investigation, Inc.

$0021-9738 / 84 / 11 / 1860 / 11 \quad \$ 1.00$

Volume 74, November 1984, 1860-1870 obtained by microcatheter from the fornices, the deepest intrarenal extensions of the pelvis, and compared with samples at the tip. Urinary osmolality was lower in the fornix, $646 \pm 106 \mathrm{mosmol} / \mathrm{kg} \mathrm{H}_{2} \mathrm{O}$, than at the tip, $1,296 \pm 99$ mosmol/ $/ \mathrm{k} \mathrm{H}_{2} \mathrm{O}(P<0.001)$. Similarly, U/P inulin was lower in the fornix, $48 \pm 14$, than at the tip, $128 \pm 12(P<0.001)$. The lower $\mathrm{U} / \mathrm{P}$ inulin in the pelvic urine is the result of either the addition of fluid to the pelvis, or the backleak of inulin across the epithelium lining the pelvis. To verify that the pelvic epithelium was impermeable to inulin, in group IVA $(n=4)$ the left renal pelvis was superfused with a solution of chemical inulin. Cumulative absorption of inulin from the left kidney was $0.15 \pm 0.08 \%$ of that superfused. Using $\left[{ }^{14} \mathrm{C}\right]$ inulin in group IVB $(n=3)$, similar results were obtained $(0.05 \pm 0.02 \%)$.

These findings indicate that in the renal pelvis, fluid is added to urine after it emerges from the collecting ducts. We suggest that reflux of hyperosmotic urine over the renal papilla creates a transepithelial gradient for the flux of water into the pelvis. A model that incorporates diffusive and convective forces for water and solute transport is proposed to account for these findings.

\section{Introduction}

We recently found that if the ureter is severed just beyond the tip of the renal papilla, urinary osmolality falls (1); this suggested that exchange of either water or solute between urine and renal parenchyma in the renal pelvis subserves urinary concentration. In 1966, Gertz et al. (2) reported evidence for exchange of water and solute across the papillary epithelium between the papilla and an artificial fluid superfusing the renal pelvis. Studies by several groups (3-7) confirmed these findings and suggested that such an exchange might play a role in the urinary concentrating mechanism. Most investigators proposed that recycling of urea from pelvic urine into the papilla helps maintain the high interstitial urea concentration of the latter. In only one of these studies, however, was the composition of 
pelvic urine actually determined (7). The purpose of this investigation was to determine whether the osmolality of pelvic urine differs from that of urine emerging from the terminal collecting ducts, and if so whether it is caused by the flux of solute or water.

\section{Methods}

Young, 70-110-g Munich-Wistar rats (Timco Breeding Laboratories, Houston, TX) of either sex were studied. On the evening before the experimental day, water was withheld from the rats. The rats were anesthetized with Inactin (BYK Gulden, Konstanz, West Germany), $100 \mathrm{mg} / \mathrm{kg}$ body wt, and prepared for micropuncture through the intact pelvic ureter of the left kidney, as previously described (1), except the ventral rather than dorsal aspect of the kidney was exposed. Throughout the experiment the animals received intravenous saline, $0.9 \%, 0.15 \mathrm{ml} / \mathrm{min}$ per $\mathrm{kg}$ body $\mathrm{wt}$. In some rats, inulin was added to maintain plasma inulin concentration between 30 and $50 \mathrm{mg} / \mathrm{dl}$.

Micropuncture (groups I and II). Two groups of animals were studied. In group I $(n=17)$, samples of urine were obtained by micropuncture through the translucent contracting pelvic ureter (1) in two places-at the papillary tip and in the pelvic space as far proximal to the tip as possible $(1.5-2 \mathrm{~mm})$, beside the base of the visible extrarenal papilla. This was designated the base site (Fig. 1). A small drop of colored oil (Kel F, 3M, 3M Center, St. Paul, MN) was injected to verify the location of the pipette tip in the pelvis. (After injection, the drop would often be swept up under the overlying kidney into the upper portion of the pelvis [see below], and then after a moment it would return. Sometimes this back-and-forth movement of the oil drop was repeated. Eventually, the drop was carried by peristalsis down the ureter. Occasionally, it would travel approximately halfway down the ureter and then be swept back up to the pelvis.) Usually, two samples from each site were collected. The sequence of punctures was alternated and the osmolality of each sample was determined.

In group II $(n=24)$, the same procedure was performed, except that in 15 rats (group IIA), the inulin concentration as well as osmolality was determined in samples of sufficient volume. In the other nine rats (group IIB), a sample was also obtained from the bladder catheter of final urine from the left kidney before and after the fluid samples were obtained from the pelvis. The right ureter was catheterized separately. In group IIB, the osmolality of all samples was determined; in samples sufficiently large, the concentrations of inulin and urea were also measured.

Microcatheterization (group III). A 1-ml polypropylene pipette (Nalgene Labware Div. Nalgel Sybron Corp., Rochester, NY) was heated over an alcohol burner and pulled to an outer diameter of 75$100 \mu \mathrm{m}$. The tip was threaded through a pulled-glass pipette so that the catheter protruded $\sim 1 \mathrm{~cm}$ beyond the narrow end of the glass pipette. The distal end of the catheter was cut flush with the distal end of the pipette. The outer surface of the catheter and the inner wall of the narrow end of the glass pipette were sealed together with Permabond 910 (Permabond International Division, Englewood, NJ) and allowed to dry for at least $24 \mathrm{~h}$. Just before insertion of catheter, colored oil was aspirated into the catheter.

Rats were prepared as described above. A polyethylene catheter was inserted into the right ureter. The left kidney was exposed through a ventral incision and placed in a warmed lucite cup. A fiberoptic light guide was positioned below the renal pelvis, which enabled the extrarenal papilla to be seen through the ureter by transillumination. A small incision was made in the ureter with microdissecting scissors (Roboz Surgical Instrument Co., Inc., Washington, DC) $0.5-1.5 \mathrm{~cm}$ below the tip of the papilla. Bleeding was controlled by applying Gelfoam (Upjohn Co., Kalamazoo, MI). The polypropylene catheter was inserted through the incision and gently guided between papilla and pelvic wall into the deepest recess of the pelvis, the fornix, by using a micromanipulator (Fig. 2). The catheter was left in place for 5-15 min to collect urine and then carefully withdrawn. Within a few minutes, another polypropylene catheter was inserted through the same incision and guided to a position just below the tip of the renal papilla. Urine was collected for 5-10 min. Gentle aspiration was sometimes necessary to ensure an adequate volume. The procedure

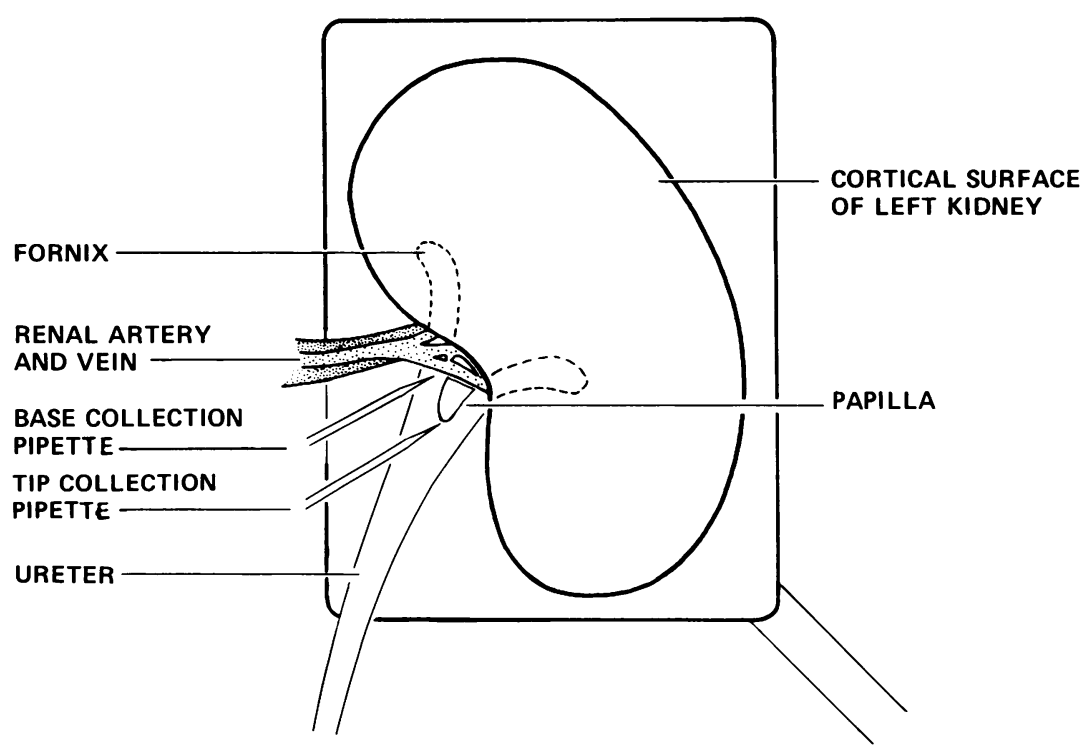

Figure 1. Pipette placement in pelvis. Micropuncture through the intact contracting ureter, groups I and II. One pipette was located adjacent to the collecting ducts at the tip of the papilla. The other pipette was inserted as far proximally as possible in the pelvic space beside the base of the visible papilla. 


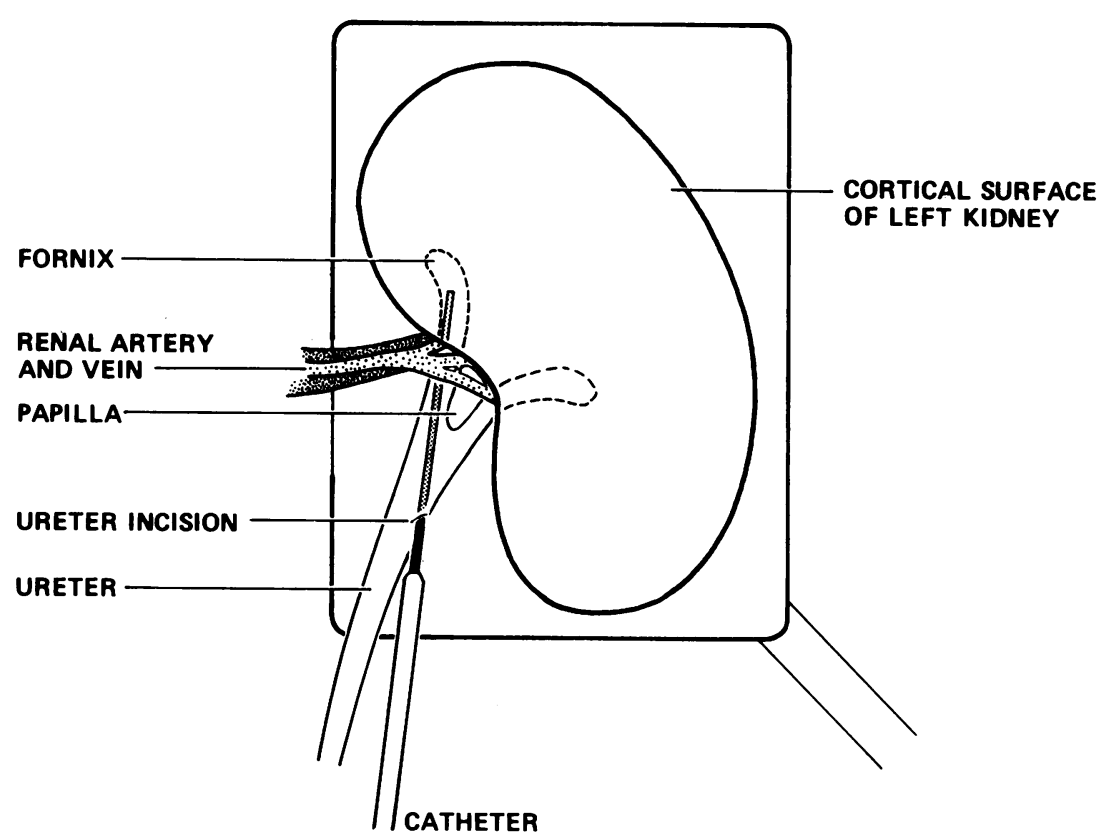

Figure 2. Catheter placement in fornix. Microcatheterization, group III. A small incision was made in the ureter and the microcatheter guided between papilla and pelvic wall into the fornix. After the catheter was withdrawn, another catheter was inserted up to the tip of the papilla (not shown). was repeated to obtain a second pair of samples from fornix and tip. Since a gradual decline in urinary osmolality sometimes occurred during the course of a microcatheterization experiment, paired samples were always collected from the fornix first so as not to bias the results toward fornix hypoosmolality. All samples were immediately placed under oil. Throughout the microcatheterization, urine was collected from the right kidney.

To check the accuracy of catheter placement, a catheter was inserted into the fornix in two rats and the pelvis was ligated to retain the catheter in situ. The kidney was removed and fixed for $24 \mathrm{~h}$ in glutaraldehyde. Subsequent dissection revealed the catheter to be in the fornix.

Superfusion of the pelvis (group IV). To determine if the pelvic epithelium is permeable to inulin, the pelvis was superfused in seven rats. Rats were anesthetized and the left kidney was exposed. The pelvic superfusion method of Bonventre et al. (5) was used. An incision was made in the left ureter $1-2 \mathrm{~cm}$ below the tip of the renal papilla. A polyethylene (superfusion) catheter was inserted through the incision to a site immediately beneath, but not in contact with, the tip of the papilla. A second (collection) catheter was inserted through the same incision so that its tip was adjacent and $5 \mathrm{~mm}$ caudad to the superfusion catheter. The catheters were anchored in situ by ligature and the ureter was tied below the incision.

The superfusion catheter was connected to an infusion pump (Harvard Apparatus Co., Inc., Millis, MA) and the left renal pelvis was superfused for $90 \mathrm{~min}$ at $0.03 \mathrm{ml} / \mathrm{min}$. The composition of the superfusate was (in millimolar): $\mathrm{NaCl}, 50 ; \mathrm{KCl}, 100 ; \mathrm{CaCl}, 2$; and $\mathrm{MgCl}, 25$. Lissamine green (3\%) was added to make the solution visible inside the pelvis and to detect leaks. In four experiments, inulin, $10 \mathrm{~g} / \mathrm{dl}$, was added to the superfusate. The osmolality of urine from the left kidney was determined before superfusion. Urea was added to the superfusate to insure that it was slightly hyperosmotic to the urine. The average urea concentration was $1,500 \mathrm{mM}$.

Before the inulin superfusion began, the urine was consistently found to have a small but detectable amount of anthrone-reactive (i.e., inulinlike) substance. It was therefore necessary to subtract a blank reading from all inulin determinations. Because of uncertainty of the true value of the correction during the inulin superfusion, we repeated the experiment in three rats with $\left[{ }^{14} \mathrm{C}\right]$ inulin (New England Nuclear, Boston, MA) $(10 \mu \mathrm{Ci})$ instead of chemical inulin. To insure that the $\left[{ }^{14} \mathrm{C}\right]$ inulin was of uniform size, i.e., that it did not contain radiolabeled fragments of smaller molecular weight that might penetrate the pelvic epithelium, the $\left[{ }^{[4} \mathrm{C}\right]$ inulin was subjected to chromatography. A column containing Sephadex G-25 (Pharmacia, Uppsala, Sweden) was washed with 4 vol of normal saline. A $0.75-\mathrm{ml}$ aliquot of $\left[{ }^{14} \mathrm{C}\right]$ inulin was placed on the column, eluted with normal saline, and collected in 0.5$\mathrm{ml}$ fractions. A homogenous peak of radioactivity was eluted in the void volume. No other peaks were seen in subsequent fractions, indicating the absence of smaller radiolabeled fragments.

Throughout the superfusion with chemical inulin, urine was collected from the right kidney. In the radioisotope experiments the right urine continued to be collected after the superfusion until the urinary radioactivity had reached undetectable levels.

The amount of inulin absorbed across the left pelvic epithelium during the superfusion (cumulative absorption, in percent) was calculated as:

Cumulative absorption

$=\left(\frac{\text { amount of inulin excreted from right kidney } \times 2}{\text { amount of inulin superfused in left renal pelvis }}\right) \times 100$.

The recovery of superfused inulin from the left renal pelvis (in percent) was calculated as:

Recovery $=\left(\frac{\text { amount of inulin excreted from left kidney }}{\text { amount of inulin superfused in left pelvis }}\right) \times 100$.

That cumulative absorption as defined in Eq. 1 is a sensitive measurement of backleak of inulin from the left pelvis is verified in Appen$\operatorname{dix}$ A. 
Analyses. Osmolality and the chemical inulin concentration of plasma and urine were determined as previously described (1). The urea concentration was determined by the method of Vurek and Knepper (8). To check the method, the urea concentration of rat urine was determined before and after addition of known amounts of urea. The average \pm SD of 10 consecutive recoveries was $101 \pm 0.82 \%$.

The $\left[{ }^{14} \mathrm{C}\right]$ inulin radioactivity was determined with a Tri-Carb 2425 liquid scintillation counter (Packard Instrument Co., Inc., Downers Grove, IL) on aliquots of superfusion fluid, plasma, and diluted urine in a modified Bray's solution. Each sample was counted to achieve a minimum of 4,000 counts and $50 \mathrm{cpm}$ above background.

Statistical analysis. Values of samples from each site were averaged and treated as one value per site per animal. All samples were used to calculate average osmolality. Approximately one-quarter of all samples in groups IIA and IIB were too small for inulin determination (group IIA) or inulin and urea determination (group IIB). The mean ratio of urine-to-plasma $(\mathrm{U} / \mathrm{P})^{1}$ osmolality was calculated using only those values in which $U / P$ inulin and U/P urea ratios were also determined. Data were analyzed by using the $t$ test for paired comparisons and expressed as the mean \pm SE (9).

\section{Results}

Micropuncture. In group I the osmolality of urine at the base of the extrarenal pelvis, $880 \pm 97 \mathrm{mosmol} / \mathrm{kg} \mathrm{H}_{2} \mathrm{O}$, was substantially lower than that at the tip of the papilla, 1,425 \pm 104 $(P<0.005)$. In group II, A and B combined (Fig. 3), osmolality at the base was $1,265 \pm 87 \mathrm{mosmol} / \mathrm{kg} \mathrm{H}_{2} \mathrm{O}$ and $1,853 \pm 69$ mosmol/ $\mathrm{kg} \mathrm{H}_{2} \mathrm{O}$ at the tip $(P<0.001)$. The $\mathrm{U} / \mathrm{P}$ inulin at the base, $192 \pm 25$, was less than $U / P$ inulin at the tip, $306 \pm 16$ $(P<0.001)$. These results imply that pelvic urine is dilute compared with tip urine because of the net addition of a hypoosmotic solution.

In group IIB, the U/P osmolality was lower at the base, $3.86 \pm 0.54$, compared with the tip, $6.01 \pm 0.31(P<0.02)$, but was also lower in the bladder, $5.27 \pm 0.25$, than at the tip $(P$ $<0.02$ ). The mean U/P inulin was lower at the base, $218 \pm 49$, than at the tip, $304 \pm 16$ (the difference, however, is not statistically significant), but was similar at the tip and in the bladder, $319 \pm 20$. The U/P urea was $59 \pm 10.6$ at the base, $98 \pm 9.4$ at the tip $(P<0.05)$, and $81 \pm 6.5$ in the bladder $(P$ $<0.005$, compared with the tip value). The fraction of filtered urea at the tip, $34 \pm 3.7 \%$, was greater than that in bladder urine, $28 \pm 3.1 \%(P<0.05)$.

Microcatheterization. Group IIIA was infused with chemical inulin $(n=8)$. Urinary osmolality was $646 \pm 106 \mathrm{mosmol} / \mathrm{kg}$ $\mathrm{H}_{2} \mathrm{O}$ in the fornix and $1,296 \pm 99 \mathrm{mosmol} / \mathrm{kg} \mathrm{H}_{2} \mathrm{O}$ in the tip $(P<0.001)$ (Fig. 4). Corresponding values for $U / P$ inulin were $48 \pm 14$ in the fornix and $128 \pm 12$ at the tip $(P<0.001)$ (Fig. $5)$. The U/P urea in the tip, $46 \pm 4$, was higher than in the fornix, 17 $\pm 5(P<0.001)$ (Fig. 6).

During catheterization of the left renal pelvis, osmolality of urine from the right kidney did not change significantly

1. Abbreviations used in this paper: $\mathrm{J}_{\mathrm{v}}$, net entry of fluid per unit surface area; $U / P$, urine-to-plasma.

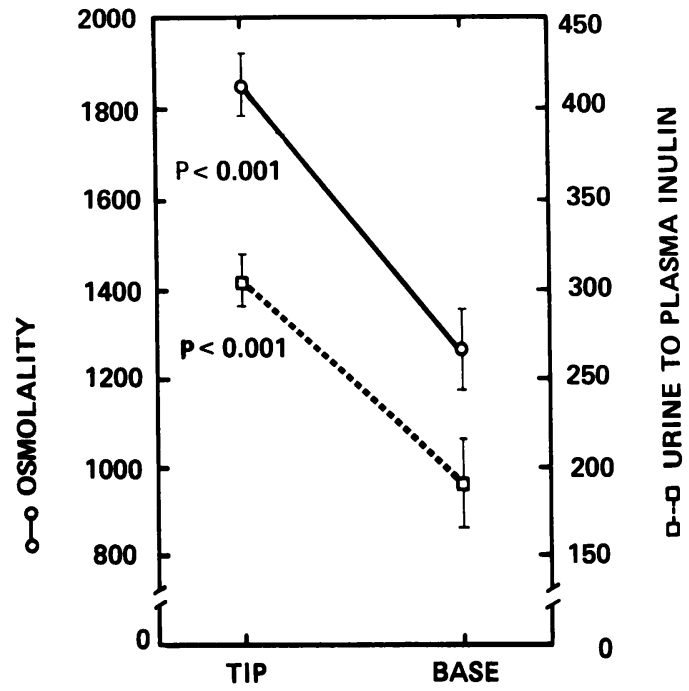

Figure 3. Urinary osmolality (mosmol/ $/ \mathrm{kg} \mathrm{H}_{2} \mathrm{O}$ ) (solid line) and U/P inulin (dashed line) at the tip and beside the base of the extrarenal papilla, group II. The vertical bars denote $\pm \mathrm{SE}$.

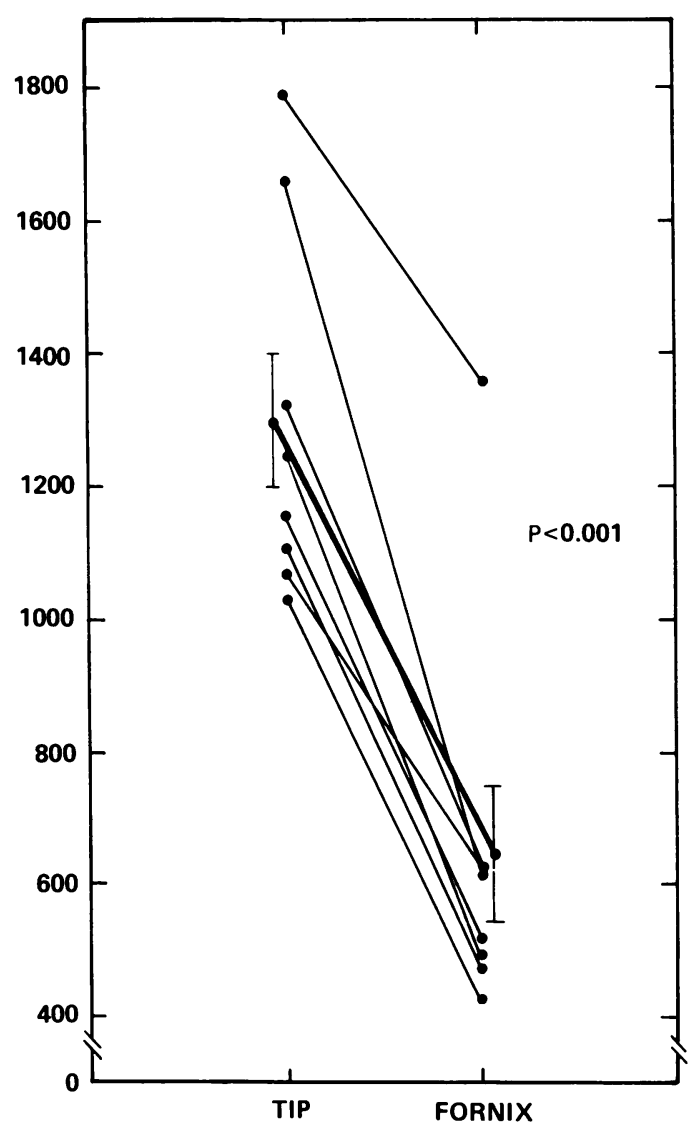

Figure 4. Urinary osmolality (mosmol/kg $\mathrm{H}_{2} \mathrm{O}$ ) at the papillary tip and in the fornix, group IIIA. Each line represents one animal. The heavy line depicts the mean; the vertical bars denote $\pm \mathrm{SE}$. 


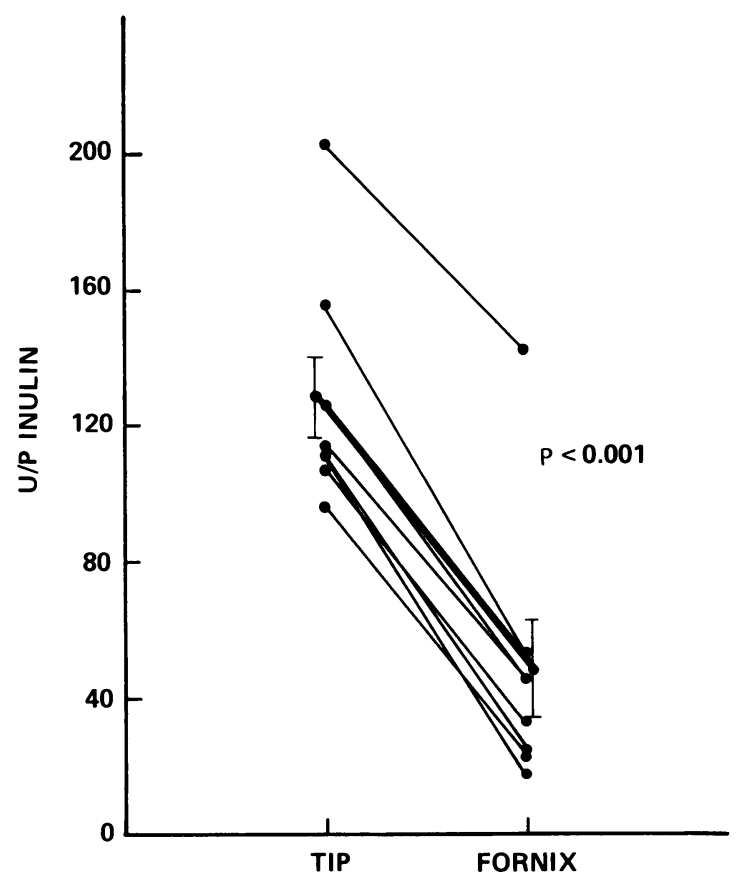

Figure 5. The U/P inulin at the papillary tip and in the fornix, group IIIA. See legend to Fig. 4.

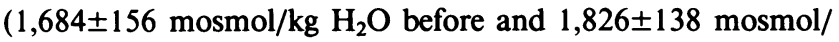
$\mathrm{kg} \mathrm{H}_{2} \mathrm{O}$ during catheterization). Glomerular filtration rate of the right kidney $(479 \pm 62 \mu \mathrm{l} / \mathrm{min})$ did not differ from that of the left kidney $(469 \pm 57 \mu \mathrm{l} / \mathrm{min})$ before catheterization and did not change significantly during catheterization of the left kidney $(536 \pm 75 \mu \mathrm{l} / \mathrm{min})$. (Urine flow from left kidney could not be measured during catheterization because of leakage from the ureteral incision.)

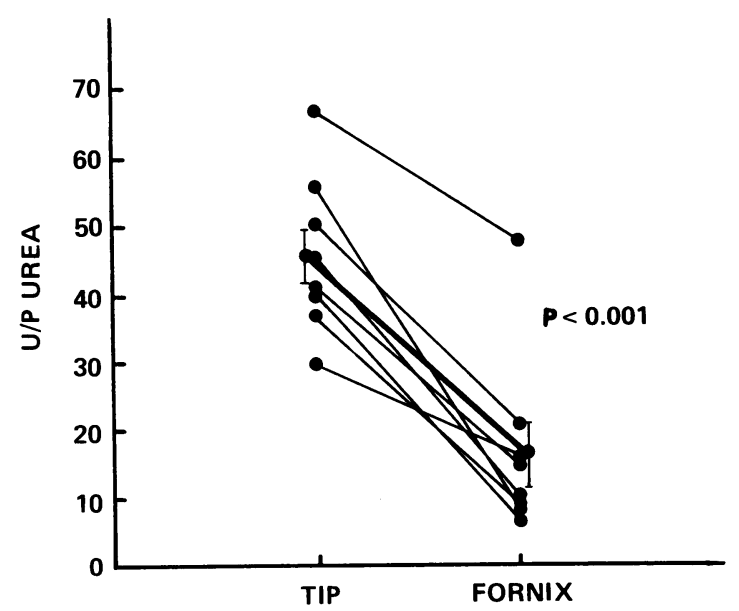

Figure 6. The U/P urea at the papillary tip and in the fornix, group IIIA. See legend to Fig. 4.
After it was demonstrated that the pelvic epithelium was impermeable to $\left[{ }^{14} \mathrm{C}\right]$ carboxyl inulin (see below), the microcatheterization experiments were repeated in group IIIB using a $\left[{ }^{14} \mathrm{C}\right]$ inulin infusion $(n=3)$. Mean osmolality of urine retrieved from the fornix, $737 \pm 78 \mathrm{mosmol} / \mathrm{kg} \mathrm{H}_{2} \mathrm{O}$, was lower than that of urine at the papillary tip, $1,324 \pm 79 \mathrm{mosmol} / \mathrm{kg}$ $\mathrm{H}_{2} \mathrm{O}$, as was $\mathrm{U} / \mathrm{P}\left[{ }^{14} \mathrm{C}\right]$ inulin: $130 \pm 55$ in the fornix vs. $296 \pm 106$ at the tip. The ratio of $\mathrm{U} / \mathrm{P}\left[{ }^{14} \mathrm{C}\right]$ inulin in the fornix to $\mathrm{U} / \mathrm{P}$ $\left[{ }^{14} \mathrm{C}\right]$ inulin at the tip was approximately the same as the corresponding ratio for chemical inulin.

Superfusion of the pelvis (group IV; $n=7$ ). To determine whether the lower inulin concentration in fornix urine is the result of inulin absorption (backleak) across the pelvic epithelium, the left renal pelvis in group IVA $(n=4)$ was superfused with a solution of chemical inulin. During the superfusion, urine from the right kidney was collected and analyzed for inulin. The cumulative absorption of inulin was $0.15 \pm 0.08 \%$. That this is an insignificant amount of inulin absorption is emphasized by the recovery of inulin in urine from the superfused pelvis, $105 \pm 4.1 \%$.

In group IVB $(n=3)$, superfusion studies were repeated using isotopic inulin instead of chemical inulin so that the analysis was not complicated by a positive urine blank reading before superfusion. Cumulative absorption was $0.05 \pm 0.02 \%$; recovery of inulin in urine from the left kidney was $106.7 \pm 2.7 \%$. From these values for cumulative absorption, the average inulin backleak could not exceed $0.23 \%$ (see Appendix A).

\section{Discussion}

The results demonstrate that in the antidiuretic rat, urine refluxing over the papilla becomes diluted by the net addition of fluid, presumably from the medullary interstitium. Since the epithelium lining the pelvis is impermeable to inulin, fluid must have been added to the urine after it emerged from the final collecting ducts at the papillary tip. We propose that as urine refluxes over the papilla, a difference in effective osmotic pressure is generated between urine and papillary interstitium, favoring net entry of fluid from interstitium to pelvis $\left(J_{v}\right)$ (Fig. 7). As the diluted urine continues to reflux toward the fornix, the transepithelial difference in osmotic pressure is sustained because of the continued decline in the axial osmolality of the papillary interstitium, and further fluid entry $\left(J_{v}\right)$ occurs. The total fluid added to the pelvis, $q,(x=0)$ (Fig. 7) becomes part of the final urine. This means that urine flowing out of Bellini's ducts at the papillary tip has not attained its final composition but is subsequently modified in the renal pelvis. ${ }^{2}$

Two potential artifacts should be considered. First, the above interpretation depends on the assumption that the pelvic epithelium is impermeable to inulin. Bonventre and colleagues (5) demonstrated that the epithelium lining the pelvis is impermeable to inulin except when epithelial integrity is

2. All the collecting ducts in the rat papilla empty at the tip of the renal papilla in the area cribrosa. 


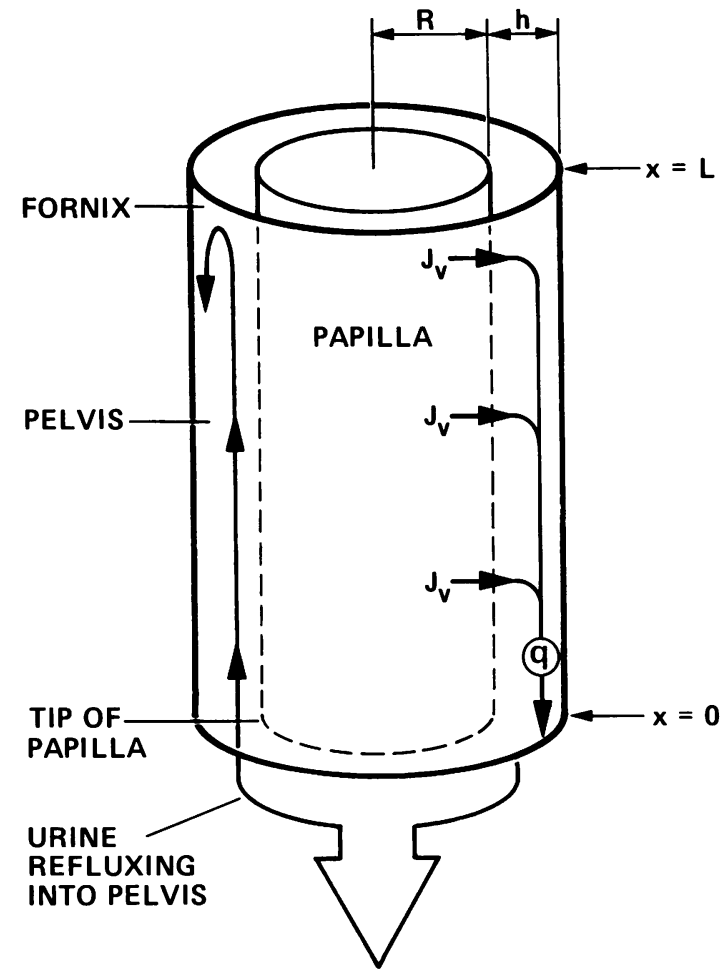

Figure 7. Model of fluid fluxes in the mammalian renal pelvis. The papilla is considered as a cylinder inside a larger cylinder, the pelvis. The space between the two concentric cylinders represents the pelvic space. On the left side, the reflux of urine leaving the collecting ducts back into the pelvis to the fornix is depicted. On the right side, the net fluid entry resulting from the reflux is shown. The hyperosmotic urine that has refluxed into the pelvis generates a transepithelial osmotic gradient for the flux of fluid, $J_{v}$, from papillary interstitium to pelvic space. This fluid creates a flow, $q$, from fornix to papillary tip. For further details, see Appendix B.

disrupted by the superfusion of unphysiologically high concentrations of $\mathrm{NaCl}$. In the present superfusion experiments (group IV), the amount of inulin absorbed from the pelvis was negligible, confirming Bonventre's observations. Second, microcatheterization may have adversely affected the process being studied. Incision of the left ureter was often followed by a fall in urinary osmolality without a decline in osmolality of urine from the right kidney, indicating a local effect of the ureteral incision. The impairment of urinary concentration resembles that observed when the ureter is completely excised beyond the tip of the papilla (1). In view of the fact that the same dilution of pelvic urine was demonstrated by micropuncture through the intact ureter, a procedure that did not impair urinary concentration, the dilute fluid in the fornix is unlikely to be an artifact of the microcatheterization technique.

To estimate the magnitude of the volume of fluid added per surface area of the pelvic epithelium $\left(\mathrm{J}_{\mathrm{v}}\right)$, a model of a simplified renal pelvis was formulated (Appendix B) to describe the decline in inulin concentration in pelvic urine (Eq. 16B in Appendix B):

$\frac{c}{c_{0}}=\exp \left\{\left[\frac{2 R \mathrm{~J}_{\mathrm{v}} L^{2}}{\mathrm{D}\left(h^{2}+2 R h\right)}\right] \frac{x}{L}\left[\frac{x}{2 L}-1\right]\right\}$,

where $c$ is the urinary concentration of inulin in the pelvis at any point $x$ from the tip of the papilla $(x=0)$ to the fornix $(x=\mathrm{L}) ; c_{0}$ is the urinary concentration of inulin at the tip of the papilla; $R$ is the radius of the papilla; $J_{v}$ is the net entry of fluid per unit surface area; $L$ is the length of the pelvis; $\mathrm{D}$ is the free diffusion coefficient for inulin (11); and $h$ is the width of the pelvic space. Eq. 3 was solved for $J_{v}$ (Appendix B). In deriving Eq. 3, it was assumed that (a) the pelvic epithelium is impermeable to inulin; $(b) \mathrm{J}_{\mathrm{v}}$ is constant everywhere in the pelvis and results in a flow of fluid, $q$, from fornix toward the papillary tip; and $(c)$ inulin diffuses freely from the papillary tip toward the fornix. In this initial attempt, only steady state behavior was considered; thus, intermittent volume fluxes into and out of the pelvis caused by ureteral peristalsis were neglected. In brief, the equation describes the profile of inulin concentration in the pelvis $\left(c / c_{0}\right)$ as the result of a balance between the diffusion of inulin from tip to fornix and the convection of inulin from fornix to tip. It should be emphasized that this is intended as an estimate of the magnitude of the fluid volume added to the pelvis. A more precise calculation requires a model that incorporates intermittent volume flux and a more realistic geometry, and is the subject of current study.

The resulting profiles of inulin concentration in the pelvis for several values of $J_{v}$ are plotted as a function of location in the pelvis in Fig. 8. To account for the average $U / P$ inulin ratio found experimentally in the fornix (group IIIA), $\mathrm{J}_{\mathrm{v}}=4.8$ $\times 10^{-7} \mathrm{~cm}^{3} / \mathrm{cm}^{2} \cdot \mathrm{s}$ (the heavy line in Fig. 8, upper panel). From the calculated surface area of the pelvis, the rate of fluid added to the urine, $q$ (at $x=0$ ) equals $1.6 \times 10^{-2} \mu \mathrm{l} / \mathrm{min}$, which is $<1 \%$ of urinary flow from one kidney in an antidiuretic rat.

The concentration profile of solutes other than inulin may be analyzed according to Eq. 3, but it cannot be assumed the epithelium is impermeable to the solute in question. By inserting in Eq. $3 \mathrm{~J}_{\mathrm{v}}$ calculated from the inulin concentration profile and the appropriate value for $D$, the free diffusion coefficient of the solute, a concentration profile, $c / c_{0}$, can be calculated for the solute and compared with that found experimentally. Any difference between the solute concentration in fornix fluid calculated by Eq. (3) and that actually found would imply net movement of the solute into or out of the pelvis. For example, from the value for $J_{v}$, which accounted for the decline of $\mathrm{U} / \mathrm{P}$ inulin from the tip to fornix in group IIIA and the diffusion coefficient of urea at $37^{\circ} \mathrm{C}\left(1.81 \times 10^{-5}\right.$ $\mathrm{cm} / \mathrm{s})(11)$, the predicted [(U/P urea fornix $) /(\mathrm{U} / \mathrm{P}$ urea tip $)]$, is 0.88 (Fig. 8, lower panel, the heavy line). This value is considerably greater than the ratio actually found, 0.36 . If the model is valid, these findings suggest that urea is removed 

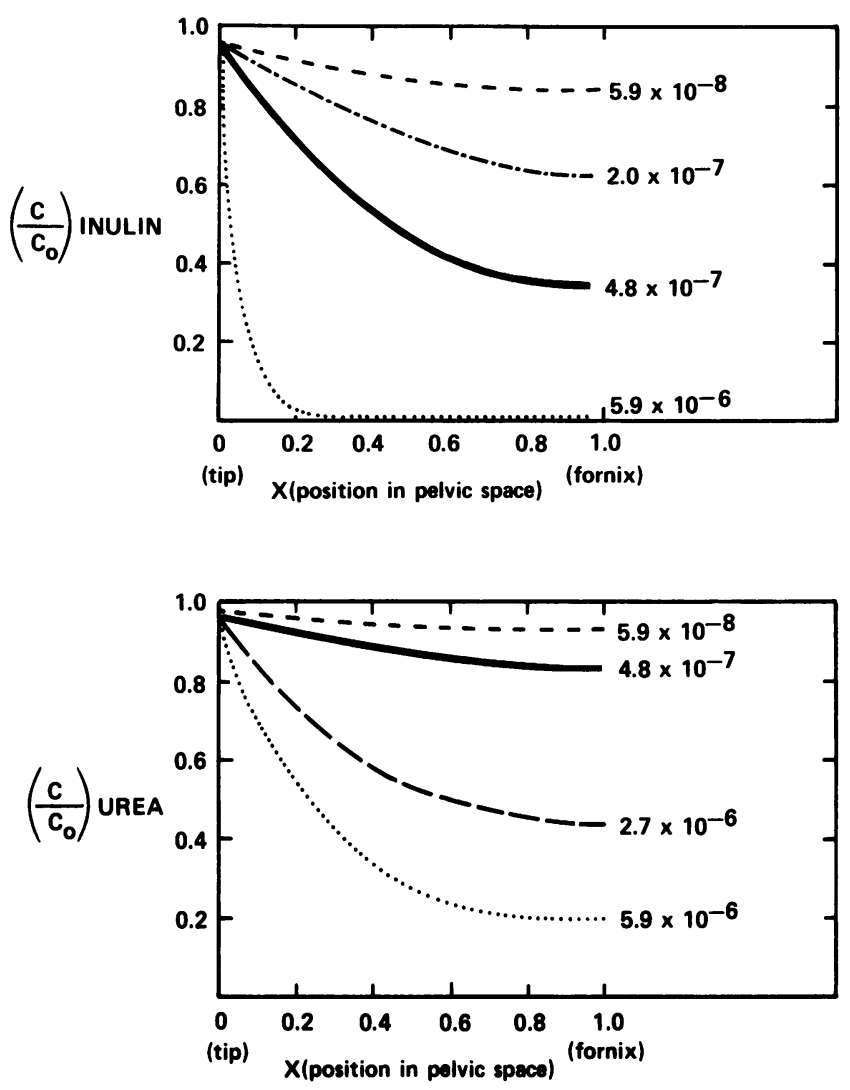

Figure 8. Inulin (upper) and urea (lower) concentration profiles in the pelvic space as a function of the dimensionless position coordinate $x$ from papillary tip $(x=0)$ to the fornices $(x=1), c$ is the concentration at $x$ and $c_{0}$ is the concentration in the tip urine. Each curve represents a different value for $J_{v}$, the volume flux (centimeters per second) from papilla to pelvic space. The heavy black line representing $\mathrm{J}_{\mathrm{v}}=4.8 \times 10^{-7} \mathrm{~cm} / \mathrm{s}$ accounts for the mean $\left[(c) /\left(c_{0}\right)\right]$ inulin in the fornices of 0.38 in group IIIA (upper) and predicts that the $\left[(c) /\left(c_{0}\right)\right]$ urea in the fornices $=0.88$ (lower). For further details see Appendix B.

from the pelvic urine, and reenters the medullary interstitium. In other words, urinary urea is recycled back to the interstitium. (The traditional approach of directly comparing the (U/P urea/ $\mathrm{U} / \mathrm{P}$ inulin) ratio at the tip with that in the fornix to determine whether urea has been removed in the fornix cannot be used, because it neglects differences in axial diffusion of inulin and urea in fluid within the pelvic space.)

Gertz and his co-workers (2) demonstrated by superfusion experiments that the papillary epithelium is permeable to urea and water. Schutz and Schnermann (3) found that the total solute concentration of the superfusate influences the osmolality of the urine and Bonventre and colleagues (5) showed that the concentration of urea in the superfusate is a particularly important determinant of final urinary osmolality. In those superfusion experiments, however, the volume flow of superfusate far exceeded the normal volume reflux of urine. In fact, Schmidt-Nielsen et al. (6) observed that full reflux does not occur at all in maximally antidiuretic rodents, although it does occur as urinary flow increases. In the present experiments, urinary reflux, observed through the intact ureter, occurred regularly, but the rats were not maximally antidiuretic (U/P osmolality = 6) so our observations are consistent with SchmidtNielsen et al.'s observations.

To judge from its structure, the epithelium lining the papilla and fornix appears capable of transcellular transport. According to Verani and Bulger (12), the epithelium lining the pelvis is composed of two types of cells-cuboidal and transitional. The cells lining the renal papilla are almost exclusively cuboidal. Their paracellular channels and apical microvilli resemble those found in other transporting epithelia. The cells undergo a change in morphology in response to antidiuretic hormone similar to that induced in the collecting duct epithelium (4). The epithelium on the opposite side of the pelvis adjacent to the renal cortex is composed mainly of transitional cells that do not appear to be conducive to transcellular transport. Between these two sites, the epithelium adjacent to the cortex and that lining the papilla, there is a gradual transition in the ratio of the two types of cell. These findings confirm the idea previously expressed by Gertz et al. (2) and later by others $(3-5,13)$ that transport in general and urea recycling in particular can occur into the renal papilla and inner medulla and, by way of secondary fornices, into the outer medulla. Whether urea normally recycles from urine to papilla, however, remains to be established. Marsh and Martin (14) could not detect a decline in mass flow of urea between the papillary tip and the ureter well below the papillary tip in the hamster.

The role of fluid addition to the renal pelvis and possibly transepithelial solute exchange between pelvic urine and medullary interstitium is not clear. There is no persuasive evidence that transepithelial exchange per se is a concentrating force. However, a mathematical model of the countercurrent system was recently published by Lory et al. (15) in which a central role was ascribed to urea recycling from pelvis to inner medulla for generation of the inner medullary longitudinal osmotic gradient. The authors predicted that pelvic urine would be found hypoosmotic to the papilla by micropuncture through the intact ureter. The ability to concentrate the urine has been observed to be related to the surface area of the pelvis (10). Animals that elaborate a very concentrated urine, such as the desert rat, Psammomys obesus, have a remarkably complex pelvis extending back to the cortex (13).

Why does removing the ureter reduce final urinary osmolality (16)? Chuang and co-workers (17) suggested that exposure of the papilla stimulates the synthesis of prostaglandin $E_{2}$, which increases papillary blood flow, causing washout of papillary solute. Reinking and Schmidt-Nielsen (18) observed 
that in antidiuresis, the papillary collecting ducts regularly open and close with a periodicity coincident with ureteral peristalsis and proposed that ureteral contraction stops flow periodically not only in the collecting ducts but in the loops of Henle and vasa recta as well. They suggested that intermittent flow facilitates the preservation of papillary hypertonicity by retarding the loss of papillary solute. Removal of the ureter would abolish intermittent flow and impair papillary hypertonicity. If the portion of the ureter overlying the papilla is paralyzed but not removed, however, urinary osmolality does not fall, even though intermittent flow in papillary structures is abolished (1). Thus, it is the intactness of the pelvic ureter rather than its contractility that is key to its contribution to papillary hypertonicity.

We propose the following working hypothesis (19). The papillary epithelium is permeable to water and certain solutes, more so in antidiuresis than in water diuresis (4). Water enters the pelvis from the papillary interstitium along a transepithelial osmotic gradient generated by refluxing urine. Solute exchange between pelvic urine and papilla occurs in a direction determined by the transepithelial concentration gradient of each permeant solute. Urea in particular reenters the papilla from the pelvic urine (i.e., recycles) since the transepithelial urea concentration gradient in antidiuresis is in that direction. Solute and water exchange is limited, however, by the small volume of urine that normally refluxes over the pelvis in antidiuresis (6). Anything that interrupts urinary reflux, such as excising the pelvic ureter (16) or severing the ureter just beyond the papillary tip ${ }^{3}$ (1) will result in loss of urea and perhaps other papillary solutes as well. Furthermore, anything that stimulates a greater reflux of urine with a reduced urea concentration, such as increasing urinary flow (6), or that mimics the same effect, such as superfusion with a urea-poor superfusate (5), will reverse the normal inward transepithelial urea gradient and cause urea to diffuse from the inner medullary-papillary interstitium into the pelvic urine. The loss of papillary solute will reduce the osmolality of the final urine. This sequence of events would facilitate the transition from

3. It is important to clarify why local paralysis of the pelvic ureter, which was done (1) to eliminate mechanical squeezing of the papilla by ureteral peristaltic contractions (20), does not compromise final urinary osmolality, while severing of the ureter just beyond the tip of the papilla does (1). As the peristalitic wave moves down the ureter, some urine is always propelled in a direction opposite to that of peristalsis (21) since the ureter contains no valves. Thus, peristalsis of the ureter beyond the tip of the papilla causes pelvic reflux of urine. As noted in Methods, urine in the ureter more than halfway to the bladder from the kidney may reflux all the way back to the fornix. Thus, urinary reflux would be abolished by severing the ureter beyond the papillary tip but not by paralysis of the pelvic ureter overlying the papilla. antidiuresis to diuresis (6). As urinary flow continues to rise, however, the intercellular spaces of cells lining the papilla would close (4); the urea and water permeability would diminish; and a new steady state of papillary hypertonicity would be reached, but at a much lower osmolality than in antidiuresis. In the reverse transition from water diuresis to antidiuresis, as urinary flow diminishes and the urea concentration in the final urine rises above that of the inner medullary interstitium, urea would recycle from pelvic urine to interstitium and increase papillary hypertonicity. Urea recycling would decline, however, as urinary flow reached its nadir and the volume of urinary reflux diminished.

Further studies will be necessary to evaluate the role of transepithelial exchange in the renal pelvis in modifying the concentration and composition of the final urine.

\section{Appendix A}

To calculate inulin absorption or backleak across the epithelium lining the renal pelvis, the left renal pelvis was superfused with inulin and the right and left urines were collected separately. To demonstrate that the superfusion experiments are sufficiently sensitive to detect inulin backleak, consider the following.

Inulin leaking across the epithelium enters the systemic circulation and can be considered to equal an intravenous infusion of inulin at the rate of leak. The rate of change of inulin mass in extracellular fluid is

$\frac{\mathrm{d}(V \mathrm{C})}{\mathrm{d} t}=Q_{\mathrm{i}} C_{\mathrm{i}}-\mathrm{k} C$,

where

$V$ is volume of distribution of inulin (in milliliters), $Q_{i}$ is infusion rate (in milliliters per minute), $C_{\mathrm{i}}$ is concentration of inulin in infusate (in milligrams per milliliter), $\mathrm{k}$ is clearance of inulin (in milliliters per minute), and $C$ is plasma inulin concentration (in milligrams per milliliter) at time $t$ (in minutes).

Assume the volume of distribution of inulin is constant, i.e., $\mathrm{d} V / \mathrm{d} t$ $=0$, then

$\frac{V \mathrm{~d} C}{\mathrm{~d} t}=Q_{\mathrm{i}} C_{\mathrm{i}}-\mathrm{k} C$

Solving this differential equation for $C$ :

$C(t)=\frac{Q_{\mathrm{i}} C_{\mathrm{i}}}{\mathrm{k}}\left[1-\exp \left(\frac{-\mathrm{k} t}{V}\right)\right]$.

The total mass of inulin excreted in time $(t)$ is given by the product of the integral of $C(t)$ and the inulin clearance, $\mathrm{k}$.

$\mathrm{k} \int_{0}^{t} C(t) \mathrm{d} t=Q_{\mathrm{i}} C_{\mathrm{i}}\left\{t+\frac{V}{\mathrm{k}}\left[\exp \left(\frac{-\mathrm{k} t}{V}\right)-1\right]\right\}=U_{\mathrm{In}} \dot{V}$

where $U_{\mathrm{In}}$ is urinary inulin concentration (in milligrams per milliliter) and $\dot{V}$ is urinary flow (in milliliters per minute). 
To test the validity of Eq. 4A, a 107-g anesthetized rat (glomerular filtration rate $=1 \mathrm{ml} / \mathrm{min} ; V=35 \mathrm{ml}$ ) was given an intravenous infusion of inulin at $24.4 \mu \mathrm{g} / \mathrm{min}$ without a priming load. This was done to simulate backleak in one kidney of $25 \%$ of the urinary excretion rate of inulin of $100 \mu \mathrm{g} / \mathrm{min}$ (an arbitrarily chosen value). Urine was collected through a bladder catheter for the duration of the infusion, $90 \mathrm{~min}$.

From Eq. 4A the predicted total inulin excretion was $1,407 \mu \mathrm{g}$. The experimental value was $1,413 \mu \mathrm{g}$.

The total mass of inulin infused in the experiment was $24.4 \mu \mathrm{g} /$ $\min \times 90 \min =2,196 \mu \mathrm{g}$. This amount simulates the mass of inulin that leaked back across the pelvic epithelium. Since this was assumed to reflect $25 \%$ backleak, the mass of inulin superfused for $90 \mathrm{~min}$ in a superfusion experiment would have to be $4 \times 2,196=8,784 \mu \mathrm{g}$. Of the inulin excreted in the above experiment, one half $(1,413 \div 2$ $=706.5 \mu \mathrm{g})$ was excreted from each kidney. Therefore, the cumulative absorption rate $($ Eq. 1$),[(706.5 \times 2) /(8,784)] \times 100=16 \%$.

Thus, a backleak of $25 \%$ of the inulin superfusion rate resulted in a cumulative absorption rate that is readily detectable. It can be shown that the ratio of backleak/cumulative absorption, $0.25 / 0.16=1.56$, is virtually constant. In group IVA, the cumulative absorption was $0.15 \%$, indicating that the backleak was no greater than $0.15 \times 1.56=0.23 \%$.

\section{Appendix B}

\section{Part I}

The purpose of this Appendix is to derive an equation to describe the concentration profile for inulin in pelvic urine (Part I) and to estimate the order of magnitude of $\mathrm{J}_{\mathrm{v}}$ in vivo (Part II). The space between the papilla and the wall of the pelvic cavity is modeled as the space between two concentric cylinders as shown in Fig. 7. $R$ is the radius of the papilla, and $h$ is the distance between the papilla and the pelvic wall. $L$ is the length of the papilla. The axial position coordinate $x$ is defined to be zero at the tip of the papilla and equal to $L$ at the top of the fornix. $\mathrm{D}$ is the coefficient of diffusion in centimeters per second.

The walls of the papilla and the pelvic cavity are assumed to be impermeable to inulin; thus, the net flow of inulin across the $x=0$ plane must be zero under steady state conditions. Only steady state behavior is considered in this simple model. The time-dependent behavior of the inulin concentration as a result of intermittent volume fluxes into and out of the pelvis caused by peristalsis of the ureter is not considered. Radial concentration gradients are neglected. $J_{v}$ is the volume flux of water across the papillary wall; the osmotic gradient across the papillary wall is assumed to cause water movement into the pelvic cavity. For the sake of simplicity, $J_{v}$ is assumed to be constant.

The volume flow rate in the pelvic space, $q$, is defined to be positive in the negative $x$-direction. A fluid balance over a thin shell (Fig. 9) bounded by $x$ and $x+\Delta x$ gives the following equation:

$q(x+\Delta x)+\mathrm{J}_{\mathrm{v}} 2 \pi R \Delta x=q(x)$.

Rearrangement yields the differential equation:

$\frac{\mathrm{d} q}{\mathrm{~d} x}=-2 \pi R \mathrm{~J}_{\mathrm{v}}$

Integrating this equation gives the following expression for the volume flow rate:

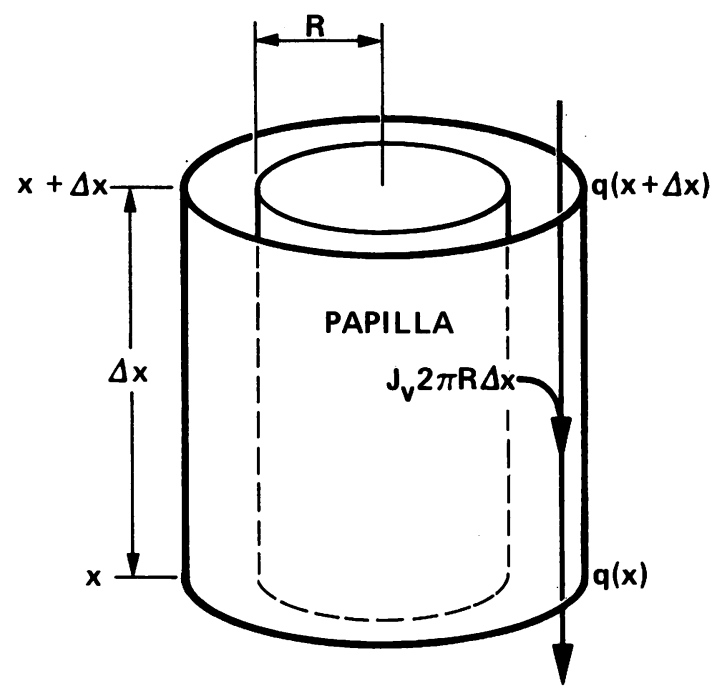

Figure 9. Mass balance of fluid in the renal pelvis. Two parallel horizontal slices of the pelvis produce a shell of thickness $\Delta x$. Fluid leaving the shell, $q(x)$, is the sum of fluid entering the shell, $q(x$ $+\Delta x$ ), and fluid added to the pelvis from the papilla, $\mathrm{J}_{\mathrm{v}} 2 \pi R \Delta x$. See Appendix B.

$q(x)=q(L)+2 \pi R \mathrm{~J}_{\mathrm{v}}(L-x)$.

Since $q=0$ at $x=L$,

$q(x)=2 \pi R \mathrm{~J}_{\mathrm{v}}(L-x)$.

Mass balance of inulin over a thin shell bounded by $x$ and $x$ $+\Delta x$ (Fig. 10) gives the following equation:

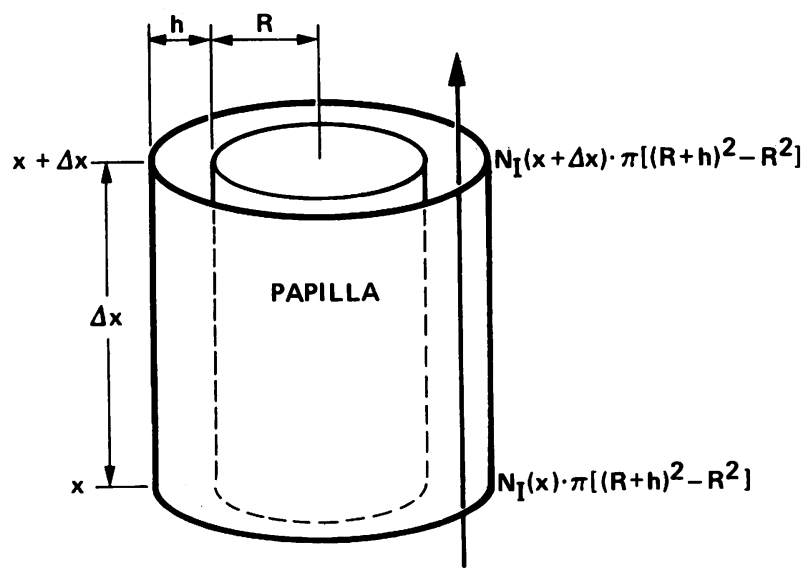

Figure 10. Mass balance of inulin in the renal pelvis. In the same shell shown in Fig. 9, inulin is neither gained nor lost from the pelvic space. The mass flow of inulin entering the shell, $\mathrm{N}_{\mathrm{I}}(x) \cdot \pi\left[(R+h)^{2}\right.$ $\left.-R^{2}\right]$, equals the mass flow of inulin leaving the shell, $\mathrm{N}_{\mathrm{l}}(x+\Delta x)$. $\pi\left[(R+h)^{2}-R^{2}\right]$. See Appendix B. 
$\mathrm{N}_{\mathrm{I}}(x) \cdot \pi\left[(R+h)^{2}-R^{2}\right]=\mathrm{N}_{\mathrm{I}}(x+\Delta x) \cdot \pi(R+h)^{2}-R^{2}$,

where $\mathrm{N}_{\mathrm{I}}$ is the inulin flux.

Since inulin is neither lost nor gained between $x$ and $x+\Delta x$,

$\frac{\mathrm{dN}_{\mathrm{I}}}{\mathrm{d} x}=0$.

The inulin flux contains a convective and a diffusive term and is defined by the following equation:

$\mathrm{N}_{\mathrm{I}}=\frac{-c q}{\pi\left(h^{2}+2 R h\right)}-\mathrm{D} \frac{\mathrm{d} c}{\mathrm{~d} x}$

Integration of Eq. $6 \mathrm{~B}$ gives

$\mathrm{N}_{\mathrm{I}}=\mathrm{C}_{1}$,

where $C_{1}$ is a constant. As stated earlier, the net movement of inulin across the $x=0$ plane is zero, so $\mathrm{C}_{1}$ in Eq. $8 \mathrm{~B}$ must be zero. Combining Eqs. 4B, 7B, and 8B gives the following differential equation for the inulin concentration:

$\frac{\mathrm{d} c}{\mathrm{~d} x}+\frac{2 R \mathrm{~J}_{\mathrm{v}}(L-x) c}{\mathrm{D}\left(h^{2}+2 R h\right)}=0$.

Nondimensionalizing Eq. 9B with $c_{0}$ (the inulin concentration at $x$ $\stackrel{0}{=}$ ), and $L$ gives

$\frac{\mathrm{d} c^{*}}{\mathrm{~d} x^{*}}+\frac{2 R \mathrm{~J}_{\mathrm{v}} L^{2}\left(1-x^{*}\right) c^{*}}{\mathrm{D} h(h+2 R)}=0$,

where $c^{*}$ and $x^{*}$ are the dimensionless inulin concentration and distance, respectively. To simplify the equation, the constant $B$ is defined to be $2 R \mathrm{~J}_{\mathrm{v}} L^{2} /[\mathrm{D} h(h+2 R)]$; Eq. $10 \mathrm{~B}$ then becomes

$\frac{\mathrm{d} c^{*}}{\mathrm{~d} x^{*}}+\mathrm{B}\left(1-x^{*}\right) c^{*}=0$.

This equation can be integrated to give

$\ln c^{*}=\mathrm{B}\left(x^{* 2} / 2-x^{*}\right)+\mathrm{C}_{2}$,

where $C_{2}$ is the integration constant. This equation is equivalent to the following:

$c^{*}=\mathrm{C}_{3} \exp \left[\mathrm{B}\left(x^{* 2} / 2-x^{*}\right)\right]$.

The constant $\mathrm{C}_{3}$ can be evaluated to be unity by applying the boundary condition $c^{*}=1$ at $x^{*}=0$. Eq. 13B then becomes

$c^{*}=\exp \left[\mathrm{B}\left(x^{* 2} / 2-x^{*}\right)\right]$

or in dimensional variables

$\frac{c}{c_{0}}=\exp \left[\mathrm{B}\left(\frac{x^{2}}{2 L^{2}}-\frac{x}{L}\right)\right]$.

Substituting for $\mathrm{B}$,

$\frac{c}{c_{0}}=\exp \left\{\left[\frac{2 R \mathrm{~J}_{\mathrm{v}} L^{2}}{\mathrm{D}\left(h^{2}+2 R h\right)}\right] \frac{x}{L}\left[\frac{x}{2 L}-1\right]\right\}$.

Part II. Calculation of $J_{v}$ to estimate in vivo results

From the morphometric data of Lacy (10), the average papillary volume is $32 \mathrm{~mm}^{3}$, and the volume of the pelvic space without the papilla is $37 \mathrm{~mm}^{3}$. The total volume, then, is $69 \mathrm{~mm}^{3}$.
If the papilla is considered as a cylinder with $L=8 \mathrm{~mm}$, then $\pi R^{2} L=32 \mathrm{~mm}^{3}$ and $R=1.1284 \mathrm{~mm}$. For the total pelvic volume, $\pi(R+h)^{2} L=69 \mathrm{~mm}^{3}$. Therefore, $h=0.5287 \mathrm{~mm}$. The diffusion coefficient $\mathrm{D}$, for inulin at $T=37^{\circ} \mathrm{C}$, is $0.240 \times 10^{-5} \mathrm{~cm} / \mathrm{s}(11)$. B $=\left[2 R \mathrm{~J}_{\mathrm{v}} L^{2}\right] /\left[\mathrm{D}\left(h^{2}+2 R h\right)\right]=4.14 \times 10^{6}\left(\mathrm{~J}_{\mathrm{v}}\right)$. Using this value for $\mathrm{B}$ and substituting different values for $J_{v}$ into Eq. 14B, a family of curves describing the concentration profile of inulin in the pelvis was calculated and is illustrated in Fig. 8.

From the in vivo data in group IIIA:

$c^{*}=\left(\mathrm{U} / \mathrm{P}\right.$ Inulin $\left._{\text {Fornix }}\right) /\left(\mathrm{U} / \mathrm{P} \operatorname{Inulin}_{\text {Tip }}\right)=48 / 128=0.38$.

We assume that the catheter tip was located on the average $\sim 80 \%$ along the length of the pelvis $(x=0.8)$. Substituting $c^{*}=0.38$ and $x^{*}=0.8$ into Eq. 14B rearranged to solve for $\mathrm{B}, \mathrm{B}=1.991$. Therefore, $\mathrm{J}_{\mathrm{v}}=4.82 \times 10^{-7} \mathrm{~cm} / \mathrm{s}$. The surface area of the papilla is given by $2 \pi r \mathrm{r}$ $=0.567 \mathrm{~cm}^{2}$. Therefore, the volume flow, $q$ at $x=0$, necessary to account for the dilution of inulin from tip to fornix $=\left(4.82 \times 10^{-7}\right.$ $\mathrm{cm} / \mathrm{s})\left(0.567 \mathrm{~cm}^{2}\right)=1.6 \times 10^{-5} \mathrm{ml} / \mathrm{min}$.

\section{Acknowledgments}

We are grateful to Mary Peterson for secretarial assistance; to Kristina Blouch, Michael Bigler, Don Chin, and Dr. R. Lane Smith for technical assistance; and to Dr. Joseph Bonventre, Dr. Paramjit Chandhoke, Dr. Floyd Rector, and Dr. Bernd Zimmerhackl for thoughtful discussions. Dr. Mark Knepper provided helpful advice concerning the microanalytic method for urea.

This work was supported by National Institutes of Health grants 5R01 AM18077 and the American Heart Association grant-in-Aid 78-875.

\section{References}

1. Oliver, R. E., D. R. Roy, and R. L. Jamison. 1982. Urinary concentration in the papillary collecting duct of the rat. Role of the ureter. J. Clin. Invest. 69:157-164.

2. Gertz, K. H., B. Schmidt-Nielsen, and D. Pagel. 1966. Exchange of water, urea and salt between the mammalian renal papilla and the surrounding urine. Fed Proc. 25:327. (Abstr.)

3. Schutz, W., and J. Schnermann. 1972. Pelvic urine composition as a determinant of inner medullary solute concentration and urine osmolality. Pfluegers Arch. 334:154-166.

4. Bonventre, J. V., M. J. Karnovsky, and C. P. Lechene. 1978. Renal papillary epithelial morphology in antidiuresis and water diuresis. Am. J. Physiol. 235:F69-F76.

5. Bonventre, J. V., R. J. Roman, and C. Lechene. 1980. Effect of urea concentration of pelvic fluid on renal concentrating ability. Am. J. Physiol. 239:F609-F618.

6. Schmidt-Nielsen, B., M. Churchill, and L. N. Reinking. 1980. Occurrence of renal pelvic refluxes during rising urine flow rate in rats and hamsters. Kidney Int. 18:419-431.

7. Schmidt-Nielsen, B., G. Rumrich, B. Sherman, and E. R. Lacy. 1977. The function of the renal pelvis in the hamster (Mesocricetus auratus). Mt. Desert Island Biol. Lab. Bull. 17:96-97. 
8. Vurek, G. G., and M. A. Knepper. 1982. A colorimeter for measurement of picomole quantities of urea. Kidney Int. 21:656-658.

9. Snedecor G. W. 1966. Statistical Methods. Iowa State University Press, Ames, IA. 85-101.

10. Lacy, E. R. 1980. The mammalian renal pelvis. Physiological implications from morphometric analyses. Anat. Embryol. 160:131144.

11. Colton, C. K., K. A. Smith, E. W. Merrill, and J. M. Reece. 1970. Diffusion of organic solutes in stagnant plasma and red cell suspension. Chem. Eng. Progr. Symp. Ser. 66(99):85-100.

12. Verani, R., and R. E. Bulger. 1982. The pelvic epithelium of the rat kidney. A scanning and transmission electron microscopic study. Am. J. Anat. 163:223-233.

13. Kaissling, B., C. de Rouffignac, J. M. Barrett, and W. Kriz. 1975. The structural organization of the kidney of the desert rodent Psammomys obesus. Anat. Embryol. 148:121-143.

14. Marsh, D. J., and C. M. Martin. 1980. Lack of water or urea movement from pelvic urine to papilla in hydropenic hamsters. Miner Electrolyte Metab. 3:81-86.

15. Lory, P., A. Gilg, and M. Horster. 1983. Renal countercurrent system. Role of collecting duct convergence and pelvic urea predicted from a mathematical model. J. Math. Biol. 6:281-304.

16. Jamison, R. L. 1970. Micropuncture study of superficial and juxtamedullary nephrons in the rat. Am. J. Physiol. 218:46-55.

17. Chuang, E. L., H. J. Reineck, R. W. Osgood, R. T. Kunau, Jr., and J. H. Stein. 1978. Studies on the mechanism of reduced urinary osmolality after exposure of the renal papilla. J. Clin. Invest. 61:633-639.

18. Reinking, L. N., and Schmidt-Nielsen, B. 1981. Peristaltic flow of urine in the renal papillary collecting ducts of hamsters. Kidney Int. 20:55-60.

19. Jamison, R. L., and W. Kriz. 1982. Urinary Concentrating Mechanism: Structure and Function. Oxford University Press, New York. 284-285.

20. Schmidt-Nielsen, B., and L. N. Reinking. 1981. Morphometry and fluid reabsorption during peristaltic flow in hamster renal papillary collecting ducts. Kidney Int. 20:789-798.

21. Shapiro, A. H., M. Y. Jaffrin, and S. L. Weinberg. 1969. Peristaltic pumping with long wavelengths at low Reynolds number. J. Fluid Mech. 37:799-825. 